\title{
Chemically selective imaging and spectroscopy with Scanning Transmission X-ray Microscopy
}

\author{
Adam P. Hitchcock ${ }^{1}$, Martin Obst ${ }^{2}$, Tolek Tyliszczak ${ }^{3}$, Samanbir Kalirai ${ }^{1}$, Dennis Bazylinski ${ }^{4}$ \\ 1. Dept. of Chemistry \& Chemical Biology, McMaster University, Hamilton, Canada \\ ${ }^{2}$ Center for Applied Geoscience, Tuebingen University, Tuebingen, Germany \\ ${ }^{3}$ Advanced Light Source, LBNL, Berkeley, USA \\ ${ }^{4}$ School of Life Sciences, University of Nevada, Las Vegas, USA
}

Soft X-ray scanning transmission X-ray microscopy (STXM) [1] is expanding rapidly at all $3^{\text {rd }}$ generation synchrotron radiation (SR) sources. At present there are 10 microscopes at 8 facilities operating with another 6 under construction or being commissioned. Given the relatively small number of $3^{\text {rd }}$ generation SR facilities which are suitable for hosting this technique, and that there were only 3 STXMs in the world a decade ago, this is an impressive indication that the technique provides useful scientific information over a wide range of disciplines. Recently there has been a trend to expand STXM capabilities by the addition of electron detectors for surface sensitive studies [2,3], X-ray fluorescence detectors for enhanced sensitivity [4], and implementation of related techniques such as ptychography [5] and local area resonant and non-resonant X-ray scattering [6]. Thus the method is more appropriately referred to as Scanning X-ray Microscopy (SXM). SXM imaging and the associated near edge X-ray absorption (NEXAFS) spectroscopy provides speciation, and quantitative mapping of chemistry and orientation (both geometric and magnetic) in 2-d and 3-d with sub $20 \mathrm{~nm}$ spatial resolution. As a photonin, photon-out technique, it is well suited for in-situ materials, environmental and biological studies since soft X-rays in the water window $(250-520 \mathrm{eV})$ readily penetrate a few microns of water. In this presentation I will outline the basic instrumentation, data analysis methods, and capabilities of soft Xray STXM. I will then present two examples in the area of biological imaging.

As immobilization by an Fe(II)-oxidizing freshwater bacteria. Chronic arsenic toxicity is a problem in many parts of the world, the best known being Bangladesh. Acidovorax sp. strain BoFeN1, a nitratereducing freshwater $\beta$-proteobacteria which grows mixotrophically oxidizing Fe(II) and using acetate as the organic co-substrate, is unaffected by As(V) concentrations as high as $10 \mathrm{mM}$. The mechanism of As accommodation or detoxification is the target of this STXM study. Two possibilities can be envisaged. The bacterial cells may take in As (which is expected through the same ion channels used for phosphorous uptake) but then continuously expel it, or sequester it inside the cell in some non-toxic form. Alternatively the As could be adsorbed on extra-cellular Fe oxides biomineralized by this species as a product of their metabolism. Initial transmission studies showed that, despite using very elevated As levels in the culture $(1 \mathrm{mM}$ of $\mathrm{As}(\mathrm{V}))$, As $2 \mathrm{p}$ signal could not be detected. However using X-ray fluorescence (XRF) detection in STXM [4] significantly improved sensitivity and lowered detection limits. As shown in Fig. 1, the absorption spectrum recorded with XRF-yield measures As $2 p$ signal at positions where the transmission spectrum is featureless. Detailed examination of a culture containing cells at various stages of growth showed that the As is primarily extra-cellular organic-rich Fe-oxides (Fig 1c), suggesting the abundant production of EPS associated with iron oxides enhances As-tolerance of the cells.

Biomagnetism. One of the most powerful capabilities of STXM is its ability to exploit the polarization as well as the photon energy properties of the X-rays. Recently we have demonstrated that STXM on a beamline with circularly polarized light, such as CLS 10ID1 or ALS 11.0.2, can measure the X-ray 
magnetic circular polarization (XMCD) of biological magnetism [7, 8]. Magnetotactic bacteria generate chains of 30-80 nm sized single crystal magnetite magnetosomes inside each cell. The collective magnetic field of the chain acts as a nano-scale compass to help the cells orient relative to the earth's field and thus navigate to the boundary of oxic and anoxic water or sediment which is their preferred ecological niche. In most cases all of the magnetosomes have a similar magnetic moment, all pointing in the same sense. Recently we have observed a number of cases where there is internal reversal - i.e. the magnetic field of one part of the chain is pointing exactly opposite to that of another part of the chain, separated by a 100-150 nm gap, the size of 1 or 2 magnetosomes (see Fig. 2). The gap region exhibits an $\mathrm{Fe}_{3}$ spectrum similar to that of magnetite but it does not have any XMCD signal [9]. It is possible these "anomalies" are situations where the magnetite biomineralization has failed or the chain is 'caught' in the act of growing. The present hypothesis is that such cases, which amounted to more than 4 $\%$ of the population of the Magnetovibrio blakemorei strain MV-1 sample studied [9], involve multiple nucleation sites where the magnetization of separate sub-chains is established before the protein system that 'assembles' the chain can bring them into sufficiently proximity that magnetic interactions of adjacent sub-chains would induce magnetization in the same direction. This work has provided insight into the biomineralization mechanisms of magnetotactic bacteria. Ongoing work on genetically modified cultures is expected to reveal further insights into these fascinating bacteria [10].

\section{References:}

[1] A.P. Hitchcock, Soft X-ray Imaging and Spectromicroscopy in Handbook on Nanoscopy, eds. Gustaaf Van Tendeloo, Dirk Van Dyck and Stephen J. Pennycook (Wiley, 2012) p. 745.

[2] B. Watts, C.R. McNeill, Macromolecular Rapid Communications 31 (2010) 1706.

[3] D. Nolle, M.Weigand, G.Schütz, and E. Goering, Microscopy \& Microanalysis 17 (2011) 834.

[4] A.P. Hitchcock et al. , Environmental Science and Technology 46 (2012) 2821

[5] M. Beckers et al, Phys. Rev. Lett 107 (2011) 208101.

[6] B. Collins et al., Nature Materials 11 (2012) 536.

[7] K.P. Lam, et al. Chem. Geology 270 (2010) 110.

[8] S. Kalirai et al. Chem. Geology 300 (2012) 14.

[9] S. Kalirai et al. Public Library of Science One 8 (2013) e53368.

[10] This research is supported by NSERC, CFI, OIT and Canada Research Chair funding. CLS is supported by NSERC,CIHR, NRC and U. Saskatchewan. ALS (LBNL) is supported by BES, DoE.
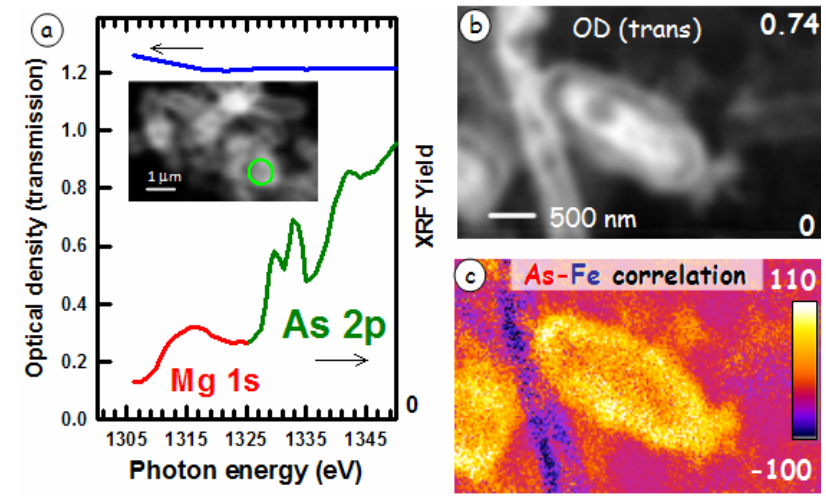

Fig. 1 (a) Transmission spectrum (blue) versus As L $\alpha$ fluorescence yield spectrum (red/green) in the region of $\mathrm{Mg} 1 \mathrm{~s}$ and As $2 p$ absorption. Insert: image of the encrusted cells; the circle indicates spectral location. (b) Image $(1346 \mathrm{eV})$ of Asrich region of BoFeN1 cells after growth in $1 \mathrm{mM} \mathrm{AsV.} \mathrm{(c)}$ Correlation of $\mathrm{As}$ (from XRF-yield) and $\mathrm{Fe}$ (from transmission). As preferentially accumulating at the outer surface of the cells, on the Fe oxide extracellular precipitates.

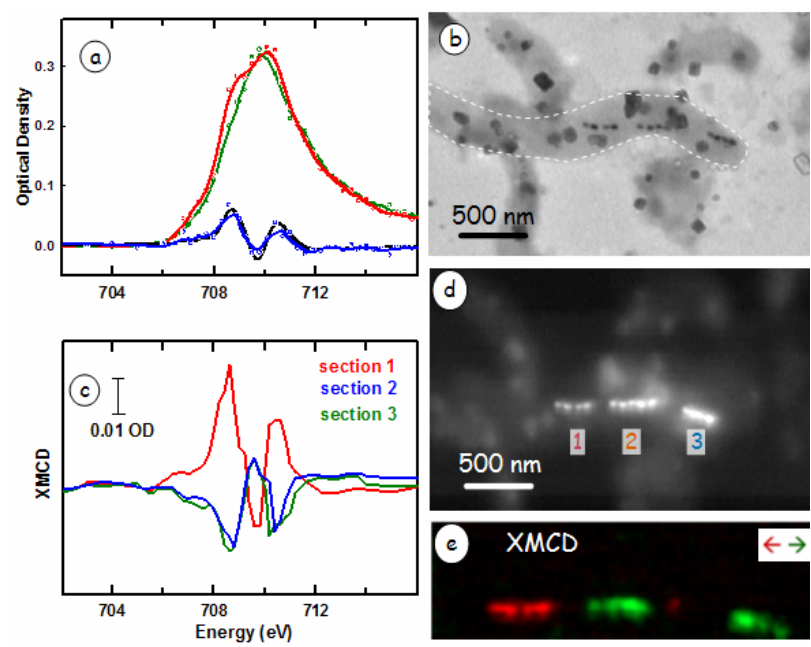

Fig. 2 (a) $\mathrm{Fe}_{3}$ STXM-XMCD of an MV-1 magnetotactic bacteria measured with circular polarization parallel (green), anti-parallel (red) to magnetic vector of chain. (b) TEM of cell with interrupted chain. (c) XMCD spectra of 3 sub-chains. (d) STXM image at $710 \mathrm{eV}$. (e) color coded XMCD signal. 